\title{
Chapter 47 \\ Report on Global Environment \\ Competitiveness of Dominican Republic
}

Dominicans is a nation on the island of Hispaniola, part of the Greater Antilles archipelago in the Caribbean region. The climate of the Dominican Republic is mostly tropical. It covers 48.3 thousand of square kilometers and has a population of 10.06 million. Its GDP reaches $\$ 55.61$ billion in 2011. Through the index system and

Score: 50.59

Rank: 60 evaluation model, the environment competitiveness index of Dominican Republic ranks at 60 in 133 countries.

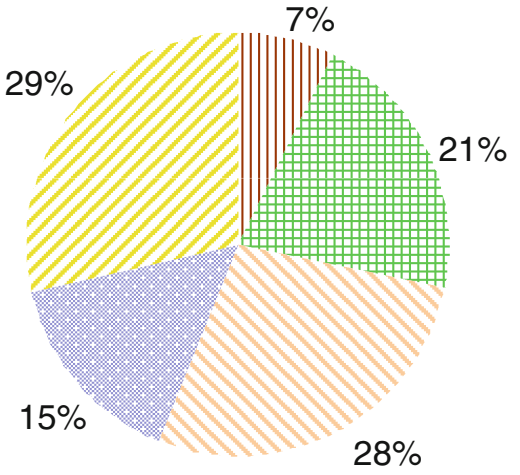

Fig. 47.1 Contribution of sub-index to GEC
III Resource Environment Competitiveness

\# Ecological Environment Competitiveness

Environment Carrying Competitiveness

Environment Management Competitiveness

Environment Harmony Competitiveness 


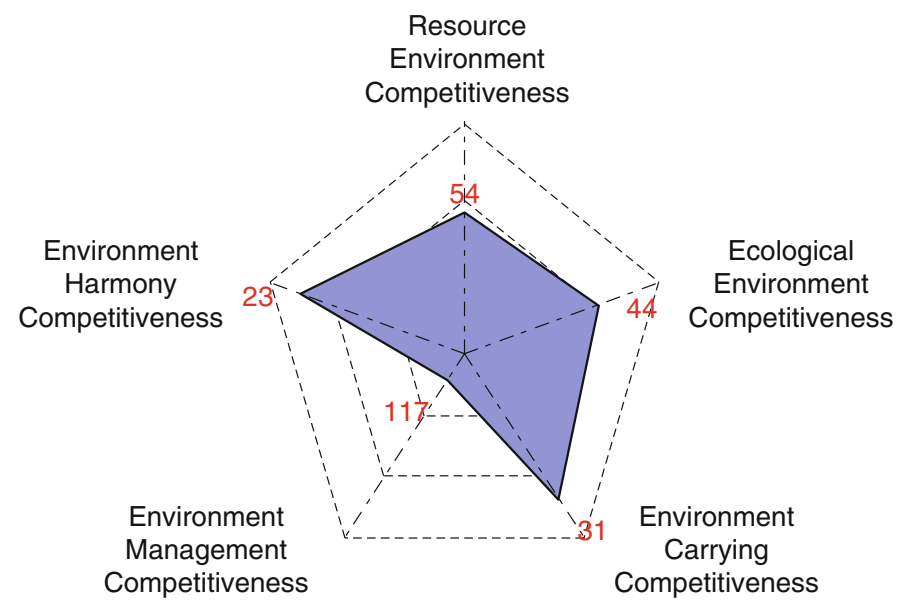

Fig. 47.2 Rank of sub-index of GEC

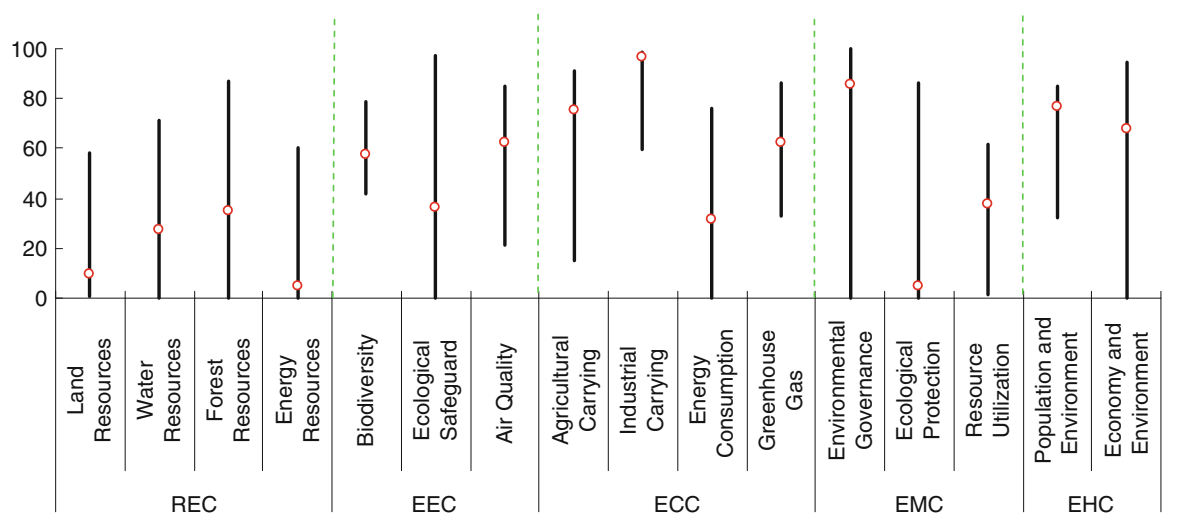

Fig. 47.3 Score and rank of the pillars of GEC

Table 47.1 Score and rank of all indicators of GEC

\begin{tabular}{lrrlrc}
\hline Indicators & Score & Rank & Indicators & Score & Rank \\
\hline $\begin{array}{l}\text { 1 Resource Environment } \\
\quad 18.64\end{array}$ & 54 & Surface water & 4.69 & 58 \\
$\quad$ Competitiveness & & & Annual precipitation & 48.15 & 38 \\
1.1 Land Resources & 9.85 & 85 & Groundwater & 34.85 & 18 \\
Land area per capita & 0.84 & 113 & Total internal renewable & 21.92 & 49 \\
$\begin{array}{l}\text { Percentage of arable land to } \\
\quad 27.98\end{array}$ & 48 & water resources & & \\
$\quad$ total land area & & & 1.3 Forest Resources & 34.64 & 45 \\
$\begin{array}{l}\text { Arable land per capita } \\
\text { 1.2 Water Resources }\end{array}$ & 3.72 & 105 & Growing stock in forest and & 50.37 & 89 \\
\hline
\end{tabular}


Table 47.1 (continued)

\begin{tabular}{|c|c|c|c|c|c|}
\hline Indicators & Score & Rank & Indicators & Score & Rank \\
\hline $\begin{array}{l}\text { Proportion of land area } \\
\text { covered by forest }\end{array}$ & 47.80 & 33 & \multirow{2}{*}{$\begin{array}{l}\mathrm{SO}_{2} \text { emissions per unit } \\
\text { of value added of } \\
\text { industry }\end{array}$} & 99.78 & 71 \\
\hline Forest area per capita & 1.37 & 74 & & \multirow{4}{*}{99.56} & \multirow{5}{*}{16} \\
\hline 1.4 Energy Resources & 5.08 & 69 & \multirow{4}{*}{$\begin{array}{l}\text { Annual freshwater } \\
\text { withdrawals for industry } \\
\text { per value added of } \\
\text { industry }\end{array}$} & & \\
\hline Fossil energy & 0.00 & 64 & & & \\
\hline Energy production & 0.20 & 114 & & & \\
\hline Proportion of combustible & 22.92 & 40 & & & \\
\hline renewable and waste to & 22.92 & & 3.3 Energy Consumption & 31.43 & 96 \\
\hline total energy consumption & & & Energy consumption per & 99.64 & 87 \\
\hline $\begin{array}{l}\text { Net energy imports of the } \\
\text { energy consumption }\end{array}$ & 2.83 & 109 & $\begin{array}{c}\text { unit of land area } \\
\text { Ratio of clean energy }\end{array}$ & 2.65 & 87 \\
\hline $\begin{array}{l}2 \text { Ecological Environment } \\
\text { Competitiveness }\end{array}$ & 52.86 & 44 & $\begin{array}{l}\text { consumption } \\
\text { Elasticity of energy }\end{array}$ & \multirow[t]{2}{*}{13.82} & 75 \\
\hline 2.1 Biodiversity & 57.41 & 78 & \multirow{3}{*}{$\begin{array}{l}\text { Elasticity of electric power } \\
\text { consumption }\end{array}$} & & \multirow[b]{2}{*}{102} \\
\hline Threatened fish species & 90.09 & 58 & & 9.59 & \\
\hline $\begin{array}{l}\text { Threatened mammal } \\
\text { species }\end{array}$ & 96.74 & 30 & & 62.09 & 62 \\
\hline Threatened plant species & 98.42 & 84 & Growth rate of $\mathrm{CO}_{2}$ & 54.61 & 51 \\
\hline $\begin{array}{l}\text { GEF benefits index for } \\
\text { biodiversity }\end{array}$ & 0.90 & 84 & \multirow{2}{*}{$\begin{array}{l}\text { emissions } \\
\text { Growth rate of Methane } \\
\text { emissions }\end{array}$} & 61.23 & 44 \\
\hline 2.2 Ecological Safeguard & 36.03 & 30 & & \multirow[b]{2}{*}{99.57} & \multirow[b]{2}{*}{90} \\
\hline Terrestrial protected areas & 60.05 & 22 & \multirow{2}{*}{$\begin{array}{l}\mathrm{CO}_{2} \text { emissions per unit of } \\
\text { land area }\end{array}$} & & \\
\hline Marine protected areas & 0.00 & 90 & & \multirow[b]{2}{*}{40.41} & \multirow{2}{*}{68} \\
\hline 2.3 Air Quality & 62.07 & 57 & $\begin{array}{l}\mathrm{CO}_{2} \text { emissions per unit of } \\
\text { energy consumption }\end{array}$ & & \\
\hline Inhalable particles (PM10) & 85.40 & 39 & \multirow{2}{*}{$\begin{array}{l}4 \text { Environment Management } \\
\text { Competitiveness }\end{array}$} & \multirow{2}{*}{38.95} & \multirow{2}{*}{117} \\
\hline Particulate matter (PM2.5) & 93.27 & 20 & & & \\
\hline $\begin{array}{l}\text { Index of indoor air } \\
\text { pollution }\end{array}$ & 33.20 & 60 & $\begin{array}{l}\text { 4.1 Environmental } \\
\text { Governance }\end{array}$ & 85.50 & 76 \\
\hline Nitrogen oxides emission & 68.53 & 37 & Agricultural chemicals & N/A & N/A \\
\hline Sulfur dioxide emission & 40.63 & 68 & regulation & & \\
\hline $\begin{array}{l}3 \text { Environment Carrying } \\
\text { Competitiveness }\end{array}$ & 70.29 & 31 & $\begin{array}{l}\text { Percentage of the rural } \\
\text { population with access to }\end{array}$ & 84.00 & 75 \\
\hline 3.1 Agricultural Carrying & 75.49 & 25 & an improved water & & \\
\hline Cereal yield per unit of & 43.59 & 35 & source & & \\
\hline & & & Percentage of the urban & 87.00 & 114 \\
\hline $\begin{array}{l}\text { Fertilizer consumption per } \\
\text { unit of arable land }\end{array}$ & 97.80 & 42 & $\begin{array}{l}\text { population with access to } \\
\text { an improved water }\end{array}$ & & \\
\hline Annual freshwater & 95.73 & 89 & source & & \\
\hline withdrawals for & & & 4.2 Ecological Protection & 5.00 & 129 \\
\hline $\begin{array}{l}\text { agriculture per unit of } \\
\text { arable land }\end{array}$ & & & $\begin{array}{l}\text { Area of plantation and } \\
\quad \text { afforestation }\end{array}$ & N/A & N/A \\
\hline 3.2 Industrial Carrying & 96.48 & 5 & Biome protect & 0.00 & 133 \\
\hline $\begin{array}{l}\text { Net exports of goods as a } \\
\text { percentage of GDP }\end{array}$ & 93.07 & 11 & $\begin{array}{l}\text { Overfishing of fishing } \\
\text { resources }\end{array}$ & 16.67 & 98 \\
\hline Electric power consumption & 93.50 & 48 & 4.3 Resource Utilization & 37.66 & 64 \\
\hline $\begin{array}{l}\text { per unit of value added } \\
\text { of industry }\end{array}$ & & & $\begin{array}{l}\text { Utilization rate of water } \\
\text { resources }\end{array}$ & 0.67 & 48 \\
\hline
\end{tabular}


Table 47.1 (continued)

\begin{tabular}{|c|c|c|c|c|c|}
\hline Indicators & Score & Rank & Indicators & Score & Rank \\
\hline $\begin{array}{l}\text { Percentage of total internal } \\
\text { renewable water resources } \\
\text { to total water resources }\end{array}$ & 64.22 & 66 & $\begin{array}{l}\text { Renewable internal } \\
\text { freshwater resources per } \\
\text { capita }\end{array}$ & 2.53 & 79 \\
\hline Percentage of agricultural & 60.35 & 49 & $\mathrm{SO}_{2}$ emissions per capita & 94.73 & 75 \\
\hline land to total land area & & & $\mathrm{CO}_{2}$ emissions per capita & 95.04 & 52 \\
\hline $\begin{array}{l}\text { Percentage of fossil fuel } \\
\text { energy consumption to }\end{array}$ & 25.40 & 65 & $\begin{array}{l}\text { Energy consumption per } \\
\text { capita }\end{array}$ & 94.48 & 45 \\
\hline total energy consumption & & & \multirow{2}{*}{$\begin{array}{l}\text { 5.2 Economy and } \\
\text { Environment }\end{array}$} & \multirow[t]{2}{*}{67.92} & \multirow[t]{2}{*}{45} \\
\hline $\begin{array}{l}5 \text { Environment Harmony } \\
\text { Competitiveness }\end{array}$ & 72.22 & 23 & & & \\
\hline 5.1 Population and & 76.51 & 33 & $\begin{array}{l}\text { Land resource utilization } \\
\text { efficiency }\end{array}$ & 0.33 & 34 \\
\hline $\begin{array}{l}\text { Environment } \\
\text { Percentage of population }\end{array}$ & 83.00 & 73 & $\begin{array}{l}\text { Sulfur dioxide emissions per } \\
\text { unit of GDP }\end{array}$ & 92.34 & 75 \\
\hline $\begin{array}{l}\text { with access to Improved } \\
\text { sanitation facilities }\end{array}$ & & & $\begin{array}{l}\text { Carbon dioxide emissions } \\
\text { per unit of GDP }\end{array}$ & 88.20 & 64 \\
\hline $\begin{array}{l}\text { Motor vehicles per } 1,000 \\
\text { people }\end{array}$ & 84.44 & 65 & $\begin{array}{l}\text { Energy consumption per } \\
\text { unit of GDP }\end{array}$ & 90.82 & 39 \\
\hline
\end{tabular}

Table 47.2 Rank distribution of the individual indicators of GEC

\begin{tabular}{|c|c|c|c|c|c|c|}
\hline Sub-index & $\begin{array}{l}\text { Number of the } \\
\text { individual } \\
\text { indicators }\end{array}$ & $\begin{array}{l}\text { Rank } \\
1-10\end{array}$ & $\begin{array}{l}\text { Rank } \\
11-30\end{array}$ & $\begin{array}{l}\text { Rank } \\
31-60\end{array}$ & $\begin{array}{l}\text { Rank } \\
61-100\end{array}$ & $\begin{array}{l}\text { Rank } \\
101-133\end{array}$ \\
\hline $\begin{array}{l}\text { Resource Environment } \\
\text { Competitiveness }\end{array}$ & 14 & 0 & 1 & 7 & 4 & 2 \\
\hline $\begin{array}{l}\text { Ecological Environment } \\
\text { Competitiveness }\end{array}$ & 11 & 0 & 4 & 5 & 2 & 0 \\
\hline $\begin{array}{l}\text { Environment Carrying } \\
\text { Competitiveness }\end{array}$ & 15 & 1 & 2 & 5 & 7 & 0 \\
\hline $\begin{array}{l}\text { Environment Management } \\
\text { Competitiveness }\end{array}$ & 10 & 0 & 0 & 2 & 4 & 2 \\
\hline $\begin{array}{l}\text { Environment Harmony } \\
\text { Competitiveness }\end{array}$ & 10 & 0 & 0 & 4 & 6 & 0 \\
\hline Total & 60 & 1 & 7 & 23 & 23 & 4 \\
\hline
\end{tabular}

Open Access This chapter is distributed under the terms of the Creative Commons Attribution Noncommercial License, which permits any noncommercial use, distribution, and reproduction in any medium, provided the original author(s) and source are credited. 